\title{
Éditorial
}

\section{Dix regards sur le dopage}

Les dix articles qui constituent ce numéro spécial interrogent la question de la prévention du dopage. Quatre champs scientifiques (sociologique, criminologique, juridique, historique) sont convoqués pour analyser ce qui pose problème, nuit, ou, au contraire, peut favoriser la lutte contre le dopage.

Tous suivent une même logique : la lutte contre le dopage ne peut se limiter à la mise en œuvre d'un arsenal coercitif qui, pour utile qu'il soit, ne permet pas à lui seul de «prévenir » le dopage. Qu'est-ce que prévenir d'ailleurs? Trop souvent, en effet, la prévention se résume à la sanction censée apeurer le sportif potentiellement déviant (Javerlhiac \& Bodin, 2012; Javerlhiac, Bodin, \& Cabagno, 2015). Il n'en est rien. Les affaires anciennes ou récentes de dopage, l'évolution des produits et méthodes utilisés témoignent de l'impuissance de la seule sanction à endiguer ce qu'il convient d'appeler un fléau (Bodin \& Sempé, 2012). Fléau car le dopage, loin d'être en régression, progresse aussi rapidement que l'efficacité des contrôles. Fléau car il ne concerne pas seulement le sport de haut niveau et/ou le sport professionnel. Il accompagne tous les niveaux de compétition ou de pratique et surtout toutes les catégories d'âge.

Le sportif n'est pas seulement «un loup pour l'homme», pour détourner la célèbre formule de Plaute. Il veut être meilleur que l'autre quel qu'en soit le coût bien sûr. Mais le dopage est aussi autre chose. Il est un viatique à la préparation, un substitut à l'absence de récupération, un moyen «normal » dans une société prophylactique où la prise de médicaments et autres psychotropes fait partie du processus normal du « Culte de la performance » (Ehrenberg, 1991). À moins qu'il ne soit tout simplement qu'un moyen comme un autre d'affirmer son appartenance au groupe ou d'oublier « la fatigue d'être soi (Ehrenberg, 1998) dans un monde sportif incertain dans laquelle la victoire d'aujourd'hui n'augure pas nécessairement, loin s'en faut, celle de demain. Pour autant, l'exigence de résultat est là, la nécessité de la performance aussi dans un monde sportif en mutation vers un autre chose qui n'est plus ni le jeu, ni le sport, ni même, peut-être, un show moderne (Vigarello, 2002). N'y-a-t'il pas, tout simplement, une certaine forme d'utopie à penser qu'une réelle politique de prévention pourrait résoudre d'un coup un seul « la tentation du dopage » (Brissoneau, 2003) ? De nombreux débats l'ont rappelé : la compétition engendre des dérives. Brohm (1993) a montré depuis fort longtemps le rôle des performances et des records dans l'augmentation des conduites dopantes.

Le dopage pose différemment un problème simple à savoir si le crime est normal? Dès 1895, Durkheim nous invitait à réfléchir à cette question à laquelle il proposait un ensemble de réponses. Détournant à notre profit cette question demandons-nous si le dopage est normal? Pour Durkheim : «le crime est... nécessaire; il est lié aux conditions fondamentales de toute vie sociale, mais par cela même, il est utile : car ces conditions [...] sont elles mêmes indispensables à l'évolution normale de la morale et du droit» (p. 72). Ces propos nous invitent tout simplement à observer, mais aussi à prévoir, les transformations de la société tout autant que de la société sportive. L'une influençant l'autre et inversement. Dans les deux cas la performance est essentielle, la prophylaxie évidente, la nécessité de paraître cruciale.

Dix articles, donc, structurent ce numéro spécial.

Le premier, écrit par Éric Péchillon, intitulé Le sportif surhomme et sous citoyen : faut-il renoncer à sa liberté individuelle pour faire du sport de compétition?, interroge la place du sportif au plan légal. Au moment où la mise en œuvre du Code mondial antidopage 2015 suppose une harmonisation des procédures et la reconnaissance de standards internationaux, il n'est pas inutile de s'interroger sur l'émergence d'un droit touchant directement à l'exercice des libertés individuelles. Un tel code oblige chaque État à modifier ses normes internes, sans pour autant renoncer à la cohérence de son système juridique. La transposition en droit interne de normes transnationales privées ne va pas sans poser problème en France, pays qui cherche à faire de la pratique du sport une mission de service public, quitte à créer une exception sportive. Il convient de s'interroger sur le principe et les conséquences du développement international d'une politique publique de lutte contre le dopage. Le sportif est-il un individu tellement exceptionnel qu'il mérite un traitement dérogatoire? En acceptant le statut de sportif, l'individu doit-il renoncer à une partie de ses droits fondamentaux? Une lutte efficace contre le dopage suppose la mise en place de moyens dérogatoires du droit commun. 
Cette recherche de la performance ne doit pas conduire à faire peser sur le sportif une présomption de triche qui le transformerait en sous-citoyen.

Dans le deuxième, Jean-Christophe Lapouble, sous le titre Le suivi à la trace : les contraintes des sportifs appartenant aux groupes cibles, aborde la question de la localisation des sportifs. Le système de localisation des sportifs mis en place par le Code mondial antidopage n'est pas sans soulever des interrogations quant à l'atteinte à la vie privé qu'il entraîne. La circulation de certaines informations, strictement personnelles, n'est pas sans risque dans certains pays. De plus, en l'état actuel du droit les recours paraissent limités et/ou insuffisants.

Avec le troisième article, L'harmonisation internationale des dispositifs de contrôle des sportifs à l'épreuve du droit communautaire, Julie Demeslay, interroge le potentiel effet liberticide de la lutte contre le dopage « La lutte antidopage devient-elle liberticide? », pouvait-on lire dans le journal L'Équipe du 24 janvier 2008. Le processus d'harmonisation internationale de la lutte antidopage a mené à la création de l'Agence mondiale antidopage (AMA) en novembre 1999. L'étude support de sa réflexion porte sur les tensions autour des dispositifs de contrôle, mis en œuvre par l'AMA, relatifs à la localisation des sportifs. Elle s'est appuyée pour ce faire sur un corpus de sources hétérogènes. Elle montre que l'application des standards internationaux de contrôle, particulièrement la mise en œuvre du logiciel ADAMS, fait émerger deux séries d'arguments qui témoignent des principes moraux choisis par les acteurs en fonction de la façon dont ils appréhendent les contraintes qui pèsent sur eux au quotidien.

Le quatrième est le fruit d'un groupe de chercheurs, Alexandra Veuthey, Christophe Jaccoud, Dominique Malatesta et Yann Hafner Yann. Lutter contre le dopage par une dépénalisation reconstructive du sportif coupable : les enjeux et les ambiguïtés du projet Windop interroge l'articulation délicate entre sanction et prévention. La lutte contre le dopage montre une tendance croissante à articuler un axe du droit, construit sur la sanction, et un axe de la régulation enraciné dans des principes d'arbitrage et de conciliation attentifs aux notions de dignité des sportifs. Les ambiguïtés de cette lutte sont visibles à travers l'analyse socio-juridique des premiers éléments de mise en œuvre du projet-pilote de lutte antidopage Windop. Ce dernier, soutenu financièrement par l'Agence mondiale antidopage (AMA) et bénéficiant de l'expertise de sociologues et de psychologues, vise depuis 2010 à réhabiliter des sportifs convaincus de dopage, à travers diverses mesures d'accompagnement destinées à reconnaître la faute, à prévenir les récidives et à reconstruire un avenir sportif.

Le cinquième, écrit par Olivier Aubel et Fabien Ohl, Le sportif en travailleur face à la lutte anti-dopage. Éléments de critique et propositions, revient sur cette question d'une prévention basée sur la répression. La lutte contre le dopage a, pour eux, principalement été structurée par une approche répressive et préventive basée sur une conception de la faute morale du sportif. L'article propose, d'une part, de questionner les limites de cette façon de penser la lutte contre le dopage et, d'autre part, d'utiliser une perspective sociologique pour réfléchir, à partir du cas du cyclisme, au rôle des conditions de travail et d'emploi des sportifs sur le recours aux produits illicites. L'analyse des conditions de travail permet d'envisager d'esquisser une autre façon de penser la réduction des risques de dopage.

Le sixième, rédigé par Christophe Brissoneau et Fabien Ohl, Effets des politiques antidopage en cyclisme sur route en 2009. Le cas de la France, questionne l'émergence de la lutte antidopage en France. Basé sur une étude, réalisée pour le compte de l'Agence mondiale antidopage, cet article se centre sur la transformation de la politique française en matière de dopage après le scandale Festina et ses effets sur la vie des cyclistes professionnels. Il interroge tout d'abord l'émergence et l'évolution des politiques antidopage françaises en cyclisme. Puis, l'article observe qualitativement les effets des politiques sur les représentations des cyclistes. Cet article est basé sur 39 entretiens avec des néo professionnels et professionnels chevronnés, ainsi que des observations ethnographiques, ceci depuis une dizaine d'années.

Mais d'où vient cette volonté de lutter contre le dopage? C'est à cette question que s'attaque Bertrand Fincoeur, dans le septième article intitulé L'instrumentalisation de l'éthique dans la lutte antidopage en cyclisme sur route. Pour l'auteur, d'abord problématisé comme un enjeu de santé à partir des années 1960, le dopage se voit rapidement condamné au nom de l'éthique sportive. L'opposition posée entre dopage et éthique est cependant le fruit d'une construction socio-culturelle qui n'a pas toujours fait sens pour les acteurs du cyclisme. Avec la multiplication des scandales de dopage à partir de la fin des années 1990, le milieu cycliste est mis sous pression par les contraintes économiques qui découlent de la révélation des scandales. Le registre de l'éthique est alors invoqué comme moyen d'organiser la survie de la profession. La mobilisation de l'éthique est toutefois purement opportuniste, d'autres types de pratiques susceptibles de porter identiquement atteinte à l'éthique du sport continuant à fleurir impunément.

De l'éthique au fair play il n'y a qu'un pas, que franchissent, dans le huitième article, Maria José Mosquera Gonzalez et Antonio Sanchez Pato, qui analysent le Modèle éducatif de la «non violence» et du fair-play pour le sport et pour la vie. Les règles éducatives à travers le Code de la «non-violence» et du fair-play. Cet article est structuré en trois parties. Dans la première sont identifiées les tendances culturelles de la société actuelle : postmodernité, décadence et résistance. Dans la deuxième, la culture de la postmodernité et de la décadence sont analysés comme responsables de l'apparition des conduites de tricherie, contraires à l'éthique. La troisième présente le modèle éducatif de la non-violence et le fair-play comme alternative pour éduquer. Afin de la rendre opérationnelle les auteurs ont développé le « Code de la non-violence et le fair-play», structuré en trois axes principaux : fair-play, compétition et coopération. 
Dans le neuvième, La politique de prévention en Espagne en question, Yannick Hernandez Bourlon-Buon et Rodrigo Pardo interrogent les pratiques préventives pour mieux en cerner les limites à travers l'exemple espagnol. Pour les auteurs, le phénomène du dopage oblige les organisations sportives à mettre en place des politiques de prévention afin de lutter contre les conduites qui en seraient à l'origine. Les orientations choisies sont analysées sous le double regard des sportifs et de leurs entraîneurs dans le cyclisme, l'athlétisme et le basket-ball. Le constat est fort. Il existe tout d'abord une méconnaissance des mesures spécifiques de prévention parmi les jeunes sportifs. Ensuite, les reportages des médias jouent un grand rôle dans la divulgation d'informations loin d'être toujours conformes à la réalité des faits. Tout cela met en situation de vulnérabilité les jeunes sportifs qui ne trouvent ni chez leurs entraîneurs ni auprès de leurs familles des informations plus précises. En conséquence les informations retenues sont floues et centrées sur la sanction plutôt que sur les effets nuisibles pour la santé ou le développement de l'aspect éthico-moral.

Enfin, restant en Espagne, Noemi Arjona-Garcia et Teresa-Gonzalez-Aja, posent dans le dixième article la question $\mathrm{du}$ corps comme pierre angulaire du recours au dopage. Dans cet article intitulé Le dopage entre performance et perception du corps en Espagne, elles montrent que le dopage, constitue non seulement une préoccupation majeure dans le monde du sport, mais aussi dans l'espace public. À des degrés différents cependant. À travers l'exemple des affaires de dopage des sportifs espagnols relatés durant quarante année dans les journaux sportifs espagnols, les auteurs analysent la manière d'en rendre compte et l'évolution de la conception du rapport corps/dopage dans le discours journalistique. Dans le premier cas, les affaires trop visibles, trop connues dérangent. Dans le second, le débat oscille entre une médiatisation basée sur le sensationnel, la défense jusqu'au-boutiste des sportifs suspectés et le fait de vilipender les tricheurs. Il s'agit là de trois étapes successives identifiées de manière récurrente à travers l'étude des journaux sportifs. Le but de ce texte est d'offrir une approche analytique de l'histoire du dopage en Espagne.

Ces dix contributions éclairent ainsi, à partir d'exemples divers et variés, pris en Belgique, en Espagne, en France et en Suisse, la difficulté à comprendre et prévenir le dopage. La lutte contre ce dernier ne peut progresser qu'à travers une approche systémique dans laquelle tous (dirigeants, entraîneurs, parents, athlètes, pairs, médecins, journalistes...) s'allient tout en respectant les libertés individuelles. Car le problème est là, la lutte contre le dopage ne peut et ne doit ni aller à l'encontre des libertés les plus fondamentales, ni laisser croire que la sanction résoudra tout.

Sophie Javerlhiac et Aude Le Saux-Slimane Université Rennes II

\section{Bibliographie}

Bodin, D., \& Sempé, G. (2012). Faut-il légaliser le dopage? Revue du M.A.U.S.S., 40, 321-334.

Brissoneau, C. (2003). La tentation du dopage. In P. Duret \& D. Bodin (dir.), Le sport en questions (p. 97-109). Paris : Chiron, coll. « Sports Études ».

Brohm, J.-M. (1993). Les meutes sportives. Critique de la domination. Paris : L'harmattan.

Durkheim, E. (1997). Les règles de la méthode sociologique. Paris : PUF, coll. «Quadrige».

Ehrenberg, A. (1991). Le Culte de la performance. Paris : Calmann-Lévy.

Ehrenberg, A. (1998). La Fatigue d'être soi - dépression et société. Paris : Odile Jacob.

Javerlhiac, S., \& Bodin, D. (2012). En guise de conclusion. Droits de l'homme et dopage : un raisonnement impensable? In D. Bodin, E. Paget, \& G. Sempé (dir.), Droits de l'homme et dopage (pp. 198-201). Strasbourg : Éditions du Conseil de l'Europe.

Javerlhiac, S., Bodin, D., \& Cabagno G. (2015). Les politiques de prévention en question. In D. Bodin, S. Javerlhiac, \& J-N, Renaud (dir.) Les jeunes sportifs face à la tentation du dopage. Se doper ou pas. Grenoble : PUG, coll. « Sport».

Vigarello, G. (2002). Du jeu ancien au show sportif. La naissance d'un mythe. Paris : Éditions du Seuil, coll. « La Couleur des idées ». 